\title{
NUMBER OF EQUILIBRIUM STATES OF PIECEWISE MONOTONIC MAPS OF THE INTERVAL
}

\author{
JÉRÔME BUZZI
}

(Communicated by Linda Keen)

\begin{abstract}
We prove a bound of the form suggested by S. Newhouse for the number of measures with maximal entropy for a piecewise monotonic map with $N$ monotonicity intervals: $4(N-1)$. More generally we consider a potential $\phi$ of bounded distortion. If $\sup \phi<P(f, \phi)$, we give an explicit bound in terms of $N$ and of the pressure.
\end{abstract}

\section{INTRODUCTION}

The topological pressure of a measurable transformation $f$ with respect to a potential $\phi$ is

$$
P(f, \phi)=\sup \left\{h_{\mu}(f)+\int \phi d \mu: \mu \in \mathscr{E}(f)\right\}
$$

where $\mathscr{E}(f)$ is the set of Borel probability measures which are invariant and ergodic, and $h_{\mu}$ is the metric entropy. Invariant and ergodic probability measures $\mu$ such that $h_{\mu}(f, \phi)+\int \phi d \mu=P(f, \phi)$ are called equilibrium states.

We consider piecewise continuous and monotonic maps of the interval (PCM) $f:[0,1] \rightarrow[0,1]$ with a finite set of critical points $c_{0}=0<c_{1}<\cdots<c_{N}=1$ such that $f_{\left(c_{i}, c_{i+1}\right)}$ is the restriction of a continuous and strictly monotonic map defined on $\left[c_{i}, c_{i+1}\right]$. For such maps, it is well known that there always exists an equilibrium state for any regular potential (see $\S 1$ ). For potentials having bounded $f$-distortion, Denker, Keller and Urbański proved in [3] that each topologically transitive subset of $f$ (TTS) supports at most one equilibrium measure, provided that $\sup \phi<P(f, \phi)$. See the appendix for an exact statement of this result extended to maps that may have homtervals and regular, maybe discontinuous potentials.

From this "local uniqueness", one can derive global results: under the same condition, to be able to support an equilibrium measure a TTS must have an entropy no smaller than $P(f, \phi)-\sup \phi>0$. In this connection, Hofbauer [4], using his "Markov diagram" (see $\S 2$ ), showed that $n_{e q}$, the number of such TTS, is finite. We prove that

$$
n_{e q} \leq\left(\frac{h(f)}{P(f, \phi)-\sup \phi}+1\right) B
$$

Received by the editors January 19, 1994.

1991 Mathematics Subject Classification. Primary 58F11, 58F03, 54H20, 28D20. 
with $h(f)=P(f, 0)$ the topological entropy of $f$ and $B$ the "number of branches of the Markov diagram" which is bounded by $2(N-1)$, respectively $(N-1)$ if $f$ is continuous (see Main theorem, $\S 3$ ). For $\phi$ identically zero, this gives $n_{e q} \leq 2 B \leq 4(N-1)$, a bound of the form suggested by $S$. Newhouse in [9].

\section{CODING AND EXISTENCE OF EQUILIBRIUM STATES}

Let $f$ be a PCM and $\phi$ a regular real function on $[0,1]$ (on every point $\phi$ admits left and right limits). We introduce two versions of this system: (i) $(I, T, \phi)$ from which the discontinuities have been removed by doubling some points; and (ii) $(\Sigma, \sigma)$ the symbolic system.

One doubles every critical point of $f$, every discontinuity point of $\phi$ and their backward orbits, and gets an extension $(I, T, \phi)$ such that $I$ is a totally ordered metric compact, $T$ and $\phi$ are continuous, and $I$ admits a natural partition $P$ into compact-open sets on which $T$ is strictly monotonic. Since only a denumerable subset of points are doubled and elsewhere $\phi$ is unmodified, there is a bijection-preserving entropy between the two sets of non-atomic invariant measures. For our purposes, we can restrict our study to $(I, T, \phi)$.

$(I, T)$ is not expansive if $f$ has homtervals or $\phi$ had points of discontinuity. But

$$
B_{\infty}(x, \epsilon):=\left\{y \in I: \forall n \geq 0 d\left(T^{n} y, T^{n} x\right)<\epsilon\right\}
$$

is a homterval for $T$ as soon as $\epsilon$ is smaller than the distance between any two elements of $P$. So $h\left(T, B_{\infty}(x, \epsilon)\right)=0$. This is asymptotic $h$-expansivity, and it implies (see [1, Chapter 20]):

Proposition 1 [8]. If $f:[0,1] \rightarrow[0,1]$ is a PCM and $\phi:[0,1] \rightarrow \mathbb{R}$ is regular, then there exists at least one equilibrium state for $f$ with respect to $\phi$.

The symbolic expansion $\gamma: I \rightarrow P^{\mathbb{N}}$ defined by $T^{n} x \in(\gamma x)_{n}$ semi-conjugates $(I, T, P) \rightarrow(\Sigma, \sigma, \Pi)$ with $\Sigma=\gamma(I), \sigma$ the shift and $\Pi=\gamma(P)$. There is a well-known total order on $\Sigma$ such that $\gamma$ is increasing and $\sigma$ is monotonic on elements of $\Pi$ and sends intervals onto intervals. $\gamma^{-1}(\alpha)$ is either a point or, for at most a denumerable number of $\alpha$, a homterval. As above there is an entropy-preserving bijection between non-atomic invariant probability measures of $(I, T)$ and $(\Sigma, \sigma)$. We remark that $\phi$ may have no natural extension on $\Sigma$.

\section{Hofbauer's Markov diagram}

We recall Hofbauer's construction and give, in notation convenient for our purposes, some of his results for the structure of the Markov diagram of a PCM.

$(\Sigma, \sigma, \Pi)$ would be Markov if, for every $D \in \Pi, \sigma(D)$ were the union of elements of $\Pi$. This is, in general, false for PCM. Even if $f$ were not onedimensional, one could always get a Markovian extension of it by enlarging $\Pi$ into $\xi$ such that

$$
A \in \xi \text { and } D \in \Pi \Longrightarrow \sigma A \cap D \in \xi
$$

where $\xi$ defines a (possibly infinite) Markov partition for the shift on the space $\tilde{\Sigma}:=\left\{\omega \in \xi^{\mathbb{N}}: \omega_{n} \rightarrow \omega_{n+1}\right\} \quad(B$ is a successor of $A$, i.e. $A \rightarrow B$ iff $T(A) \supset$ $B) .(\tilde{\Sigma}, \sigma)$ projects itself continuously onto $(\Sigma, \sigma)$. In the general case this extension is useless as, for instance, $\tilde{\Sigma}$ may consist only of wandering points. 
In the PCM case, though the projection $\pi: \tilde{\Sigma} \rightarrow \Sigma$ may be infinite-to-1, it has good properties, the main one being:

Theorem 1 (Hofbauer [4]). If $\mu$ is an ergodic invariant Borel probability measure of $(\Sigma, \sigma)$ with positive entropy, then there exists a unique ergodic invariant measure $\hat{\mu}$ on $(\tilde{\Sigma}, \sigma)$ such that $\mu=\hat{\mu} \circ \pi^{-1}$. Also $h_{\hat{\mu}}(\tilde{\Sigma})=h_{\mu}(\Sigma)$.

This property follows from the very special structure of the graph $\xi$ (we remark that it has been generalized by Keller [6]). Denote by $E$ the set of the ends of the intervals of the partition $\Pi$. To each $e \in E$ we associate a branch $\{J(e, t): t \geq 0\} \subset \xi$ where the $J$ 's are defined by $J(e, 0)=\Pi(e)$ (the element of $\Pi$ containing $e)$ and $J(e, t+1)=T J(e, t) \cap \Pi\left(T^{t+1} e\right)(t \geq 0)$. $J(e, t+1)$ is a successor of $J(e, t)$. We call it the trivial successor.

We say that $\Pi$ cuts $T J(e, t)$ when this interval is not contained in an element of $\Pi$ (and so $J(e, t)$ has several successors). We define the cutting time function $K$ by

$$
\{K(e, 0)<K(e, 1)<\cdots<K(e, n)<\cdots\}=\{t \geq 1: J(e, t) \neq T J(e, t-1)\}
$$

(if the set on the right-hand side is not infinite we complete it with $\infty$ 's).

Theorem 2 (Hofbauer [4]). The Markov extension $\xi$ of a PCM is the union of a finite number of branches, $\xi=\{J(e, t): e \in E$ and $t \geq 0\}$. There exists a map $Q: E \times \mathbb{N}^{*} \rightarrow E \times \overline{\mathbb{N}}$ so that:

(1) $K(e, n+1)=K(e, n)+K(Q(e, n+1))$.

(2) $J(e, t)$ has non-trivial successors iff $t=K(e, n)-1$ for some $n \geq 0$, and these comprise at least $J(K(e, Q(e, n)))$ and may be a number of $J(f, 0)$ with $f \in E$.

Remark. The $J(e, t)$ are not necessarily distinct. To keep things as simple as possible we freely write $J(K(r, s)-1)$ instead of $J(e, K(r, s)-1)$.

The irreducible parts of $\xi$ are the maximal sets $G \subset \xi$ with the property that for every $(C, D) \in G^{2}$ there exists a path on $\xi$ from $C$ to $D$. The subshifts $\tilde{\Sigma}(G)$ of $\tilde{\Sigma}$ defined by an irreducible part not reduced to a loop are exactly the maximal topologically transitive subsets of postive entropy. So every equilibrium measure, having positive entropy under our assumptions, is concentrated on some $\tilde{\boldsymbol{\Sigma}}(G)$. The local uniqueness result of [5] quoted in the appendix therefore reduces the problem of the multiplicity of equilibrium measure to bounding the number of TTS of entropy greater than $h(f) / r$ with

$$
r=\frac{h(f)}{P(f, \phi)-\sup \phi} \text {. }
$$

\section{EXPLICIT BOUNDS}

In this section we bound the number of irreducible parts of entropy greater than $h(f) / r$ under the assumption $h(f)>0$. Let $G \subset \xi$ irreducible.

We define the combinatorial entropy as the growth number of $N_{n}(s, G)$, the number of paths over $G$ of length $n$ starting from $s$ :

$$
H(G):=\limsup _{n \rightarrow \infty}\left(\sup _{s \in G} \frac{1}{n} \log N_{n}(s, G)\right) .
$$

Clearly, $h(\tilde{\Sigma}(G)) \leq H(G)$ with equality if $G$ is finite. 
We define the level of $D \in \xi$ to be $\operatorname{lev}(D)=\min \{t \geq 0: J(e, t)=D\}$ and lev_ $_{-}(G)=\min \{\operatorname{lev}(D): D \in G\}$.

Lemma 1. Let $\omega$ be a path on $\xi$. If $\omega_{t-1}$ has several successors in $\xi$, then for $\max (t-q, 0) \leq u \leq t-2, \omega_{u}$ has only one successor in $\xi$, where $q$ is the second highest level among successors of $\omega_{t-1}$.

Proof. If there were no identifications that the proof would be trivial, one would have $\omega_{t-1}=J(K(e, n))-1$, the second highest level would be $K(Q(e, n))$, and

$$
\begin{aligned}
J(K(e, n-1)) & \rightarrow J(K(e, n-1)+1) \rightarrow \ldots \\
& \rightarrow J(K(e, n-1)+K(Q(e, n))-1)=J(K(e, n)-1)
\end{aligned}
$$

would be the only backward path of that length from $\omega_{t-1}$.

For the general case, we endow $E \times \mathbb{N}$ with the trivial arrows $(e, n) \rightarrow$ $(e, n+1)$, the arrows defined by $Q$ (in analogy to Theorem 2$)$ and which may be arrows from $(K(e, n)-1)$ to $(g, 0)$, so that $J: E \times \mathbb{N} \rightarrow \xi$ verifies $J(\operatorname{succ}(e, n))=\operatorname{succ}(J(e, n))$. Therefore we can lift the path to $X$ where the result is clear as

$$
q \leq \operatorname{lev}(J(K(Q(e, n)))) \leq K(Q(e, n)) .
$$

Lemma 2. $H(G) \leq \log 2 / \operatorname{lev}_{-}(G)$

Proof. Let $s \in G$. We may assume $l:=\operatorname{lev}_{-}(G)>0$. Assume that $N_{n+l}(s, G)$ $>2 N_{n}(s, G)$. There exists a path of length $n$ with three distinct extensions $\omega, \omega^{\prime}, \omega^{\prime \prime}$ of length $n+l$. Let $t:=\min \left\{t \in \mathbb{N}: \omega_{t} \neq \omega_{t}^{\prime}\right.$ or $\left.\omega_{t} \neq \omega_{t}^{\prime \prime}\right\}$. If $\omega_{t}, \omega_{t}^{\prime}, \omega_{t}^{\prime \prime}$ are all distinct, then one is of zero level: of three distinct successors of any node of $\xi$, one is of level zero. This implies $\operatorname{lev}_{-}(G)=0$. Otherwise $\omega_{t}=\omega_{t}^{\prime}$ and there exists $u:=\min \left\{u>t: \omega_{u} \neq \omega_{u}^{\prime}\right\}<n+l$. By Lemma 1, $t \leq u-K(Q(f, m))$. But $J(K(Q(f, m))) \in G: \operatorname{lev}_{-}(G) \leq K(Q(e, n))$. Therefore, $l>u-t \geq \operatorname{lev}_{-}(G)$. This shows that $N_{n+\operatorname{lev}_{-}(G)}(s, \bar{G}) \leq 2 N_{n}(s, G)$ and gives the result.

Lemma 3. If $G$ is a loop, then $H(G)=0$. Otherwise $H(G) \geq \log 2 / \operatorname{card} G$.

Proof. If every node of $G$ has only one successor in $G$, then $H(G)=0$. Otherwise there exists $D_{0}, D_{1} \neq D_{2}$ with $D_{0} \rightarrow D_{1}, D_{0} \rightarrow D_{2}$ and paths $D_{0} D_{1} \cdots D_{0}$ and $D_{0} D_{2} \cdots D_{0}$ each of length less than card $G$. Let $S \subset G$ be the union of these two loops. $N_{n+\operatorname{card} G}(s, S) \geq 2 N_{n}(s, S)$. So $H(G) \geq H(S) \geq$ $\log 2 / \operatorname{card} G$.

First we recover that the entropy is strictly decreasing along branches in the unimodal case:

Theorem 3 (Hofbauer [5]). If $f:[0,1] \rightarrow[0,1]$ is unimodal (continuous, single interior maximum) and of positive entropy, then it is intrinsically ergodic.

Proof. We may assume that $f(1)=f(0)=0$. We have the following identifications: $J(1, t)=J(0, t)=J(0,0), J(c-, t)=J(c+, t)$ for $t \geq 1$. If $T c=1$, then $\tilde{\Sigma}$ is the full 2 -symbol shift and the result is clear. Assume $T c<1$. $J(1,0)$ has no predecessor, so the supports of maximal measures are to be found in $\{J(c-, t): t \geq 0\}$. 
Let $G_{0} \subset \xi$ be irreducible, of positive entropy and of minimal level. If $G_{0}$ is infinite, then it is the unique irreducible part with positive entropy. Assume $G_{0}$ is finite. If $G \subset \xi$ is any other irreducible part of positive entropy, then lev $-(G) \geq \operatorname{lev}_{-}\left(G_{0}\right)+\operatorname{card} G_{0}$, so lev $(G)>\operatorname{card} G_{0} \geq \log 2 / H\left(G_{0}\right)$ and hence $h(\tilde{\Sigma}(G)) \leq H(G) \leq \log 2 / \operatorname{lev}_{-}(G)<H\left(G_{0}\right)=h\left(\tilde{\Sigma}\left(G_{0}\right)\right)$.

We proceed now to the multimodal case.

Main Theorem. Let $B$ be the number of non-trivial branches, that is, the number of $e \in E$ such that $\{J(e, k): k \geq 0\}$ meets irreducible parts of positive entropy. The number of irreducible parts of entropy greater than $h(f) / r$ is

$$
n \leq(r+1) B \text {. }
$$

Proof. Set $l:=r \log 2 / h(f)>0$. Assume first $l \geq 1$. Denote by $\xi_{l}$ the set of vertices contained in a non-trivial branch and of level strictly smaller than $[l]$ ( [ $\cdot]$ is the integer part). We split the irreducible parts of enough entropy into two sets. The $G$ 's in the first set are those $G \subset \xi_{l}$ not reduced to a loop. By Lemma 3 , card $G \geq l / r$. The number of such $G$ is

$$
n \leq \operatorname{card} \xi_{l} /(l / r) \leq r \frac{[l]}{l} B \text {. }
$$

The remaining $G$ form the second set: $G \not \subset \xi_{l}$. By Lemma $2, \operatorname{lev}_{-}(G) \leq l$. $\{\operatorname{lev}(D): D \in G\}$ is an interval of integers. So $G$ has an element of level $[l]$. But $\operatorname{card}\{D \in \xi: \operatorname{lev}(D)=[l]\} \leq B$. The number of such $G$ is therefore less than $B$.

If $l<1$, then $H(G) \geq h(f) / r>\log 2$ implies that $G$ contains a vertex of zero level. The number of $G$ is bounded by $B$.

One can always assume that $f\{0,1\} \subset\{0,1\}$ (if necessary one extends $f$ to a larger interval and then rescales back so that this condition is fulfilled with the number of monotonicity intervals unchanged and the non-wandering set remaining essentially the same). It is then clear that 0 and 1 are trivial branches. So we have

Corollary. Let $f:[0,1] \rightarrow[0,1]$ be piecewise monotonic with $N \geq 2$ intervals of monotonicity and $\phi:[0,1] \rightarrow \mathbb{R}$ be regular with bounded $f$-distortion. Set $r=h(f) /(P(f, \phi)-\sup \phi)$. The number of equilibrium states is then bounded by $2(r+1)(N-1)$. If $f$ is continuous, the bound reduces to $(r+1)(N-1)$.

\section{APPENDIX. UNIQUENESS ON TOPOLOGICALLY TRANSITIVE SUBSETS}

Denker, Keller and Urbański proved the uniqueness of the equilibrium state within a TTS. Here we give the modifications to their proof so that the unnecessary conditions that $f$ is without homtervals and that $\phi$ is not only to be regular (see $§ 1$ ) but also continuous are removed. Still $\phi$ must verify the following property:

Definition. $\phi:[0,1] \rightarrow \mathbb{R}$ has bounded $f$-distortion iff there exists $K<\infty$ such that for all $n \geq 0$ and $x, y \in[0,1]$ such that $f^{n}$ is monotone and continuous on $[x, y]$,

$$
\left|\sum_{k=0}^{n-1} \phi\left(f^{k} x\right)-\phi\left(f^{k} y\right)\right|<K .
$$


We recall that continuity is not sufficient for uniqueness even for a subshift of finite type (see [10, Chapter 2]). There are some cases where $\left({ }^{*}\right)$ may be easily checked: if $f$ is expanding or more generally

Proposition 2. If $f$ is piecewise $C^{2}$, without homtervals or indifferent periodic points and has no sensitivity to initial conditions around critical points, that is, if for each critical point $c$

$$
\forall \epsilon>0 \exists \delta>0 \quad \forall n \geq 0\left|f^{n} B(c, \delta)\right|<\epsilon
$$

( $|$.$| is Lebesgue measure), then Hölder continuity of \phi$ implies $\left({ }^{*}\right)$ on each TTS.

We outline the (easy) proof: $\left({ }^{* *}\right)$ implies that each irreducible part of $\xi$ is finite so that each TTS is bounded away from the critical points; $f C^{2}$ implies that the diameters of $P^{n}$ restricted to a TTS decrease exponentially with $n$ by [7, Corollary 1 of Theorem 5.1]. We remark that in the S-unimodal case, the condition on $f$ is simply infinite renormalizability.

Local Unicity Theorem (adapted from [3]). Let $f:[0,1] \rightarrow[0,1]$ be a PCM and $\phi:[0,1] \rightarrow \mathbb{R}$ be regular with bounded distortion on some TTS. If the following "small potential" inequality is verified,

$$
\sup \phi<P(f, \phi),
$$

then there exists at most one equilibrium state on that TTS.

The proof in [3] uses results of Hofbauer that are valid only for this case. To overcome this difficulty one studies the symbolic system $(\Sigma, \sigma)$ instead of $(I, T)$.

Proof of the theorem. The only thing in the proof [3] that does not carry over is the existence of an atomless $\exp (P(f, \phi)-\phi)$-conformal measure on $\Sigma$, though $\phi$ may be undefined and discontinuous on a denumerable subset of $\Sigma$. For the moment we assume the

Claim. There is a sequence of sets $F_{n} \subset I$ such that:

(1) $T^{-1} F_{n} \subset F_{n+1}$ and $\operatorname{card}\left(F_{n+1}-T^{-1} F_{n}\right)$ is bounded independently of

$$
\lim _{n \rightarrow \infty} \frac{1}{n} \log \left(\sum_{x \in F_{n}} \exp \left(S_{n} \phi(x)\right)\right)=P(f, \phi) .
$$

Regularity on $[0,1]$ gives continuity on $I$; see $\S 1$. As in the proof of $[2$, Theorem 3.13] (1) implies that condition [2,(3.6)] is satisfied. Moreover $T$ is injective on the neighborhood of any point of $I$ (in the notation of [2], $\operatorname{Crit}(T)=\varnothing)$ : so [2, Proposition 3.11] applies and there is an atomless $\exp (c-$ $\phi)$-conformal measure. But the transition parameter $c$ associated to $\left(F_{n}\right)_{n \geq 0}$ and $\phi$ is, by the claim $(2), P(f, \phi)$.

Proof of the claim. $(\Sigma, \sigma)$ is expansive for some constant $\delta>0$ : there exists a sequence of maximal $(n, \delta)$-separated sets $E_{n}$ with $E_{n+1} \supset \sigma^{-1} E_{n}$. There exists a map $i: \Sigma \rightarrow I$ such that

$$
\gamma \circ i=\operatorname{Id}_{\Sigma} \text { and } i \circ \sigma=T \circ i .
$$


Notice that, if $f$ has homtervals, $i \circ \gamma \neq \operatorname{Id}_{I}$ and $i$ is not continuous. One may choose distances on $I$ and $\Sigma$ such that $d(i(\alpha), i(\beta)) \geq d(\alpha, \beta)$.

Set $F_{n}=i\left(E_{n}\right) . F_{n}$ is $(n, \delta)$-separated but may be non-maximal. To make them maximal one has to add points to cover large homtervals. It is easy to see that the number of necessary points to complete $F_{n}$ is bounded by a polynomial function of $n$. Claim (2) follows.

[3, end of the proof of Theorem 2] implies $\operatorname{card}\left(E_{n+1}-\sigma^{-1} E_{n}\right) \leq 2 N$. This proves Claim (1).

\section{ACKNOWLEDGMENT}

I would like to thank $\mathrm{Ph}$. Thieullen for acquainting me with this subject and for many useful discussions.

\section{REFERENCES}

[1] M. Denker, C. Grillenberger, and K. Sigmund, Ergodic theory on compact spaces, Lecture Notes in Math., vol. 527, Springer, Berlin, Heidelberg, and New York, 1976.

[2] M. Denker and M. Urbański, On the existence of conformal measures, Trans. Amer. Math. Soc. 328 (1991), 563-587.

[3] M. Denker, G. Keller, and M. Urbański, On the uniqueness of equilibrium states for piecewise monotonic mappings, Studia Math. 97 (1990), 27-36.

[4] F. Hofbauer, On intrinsic ergodicity of piecewise monotonic transformations with positive entropy, Israel J. Math. 34 (1979), 213-237.

[5] _ Kneading invariants and Markov diagrams, Ergodic Theory and Related Topics (Vitt) (H. Michel, ed.), Springer-Verlag, Berlin and New York, 1982.

[6] G. Keller, Lifting measures to Markov extensions, Monatsh. Math. 108 (1989), 183-200.

[7] W. De Melo, One-dimensional dynamics, IMPA, Rio de Janeiro.

[8] M. Misiurewicz and W. Szlenk, Entropy of piecewise monotone mappings, Studia Math. 67 (1980), 45-63.

[9] S. Newhouse, On some results of Hofbauer on maps of the interval, Adv. Ser. in Dynam. Sys. 9 (Nagoya, 1990), 1991.

[10] D. Ruelle, Thermodynamic formalism, Addison-Wesley, Reading, MA, 1978.

Département de Mathématiques, Université de Paris-Sud, 91405 Orsay, France

E-mail address: jbuzzi@matups.matups.fr 